\title{
UNIVERSAL FIELD EQUATIONS FOR METRIC-AFFINE THEORIES OF GRAVITY
}

\author{
Victor Tapia* \\ Departamento de Matemáticas \\ Universidad Nacional de Colombia \\ Bogotá, Colombia \\ and \\ Maximiliano $\mathrm{Ujevic}^{\dagger}$ \\ Instituto de Matemática, Estatística \\ e Computação Científica \\ Universidade Estadual de Campinas \\ Campinas, São Paulo, Brazil
}

August 13, 2018

\begin{abstract}
We show that almost all metric-affine theories of gravity yield Einstein equations with a non-null cosmological constant $\Lambda$. Under certain circumstances and for any dimension, it is also possible to incorporate a Weyl vector field $W_{\mu}$ and therefore the presence of an anisotropy. The viability of these field equations is discussed in view of recent astrophysical observations.
\end{abstract}

PACS number: 0450

Recent observational evidence, pointing to a non-vanishing value of the cosmological constant [1] and to the existence of a preferred direction [2] in the Universe, has reopened the debate on which is the 'most correct' theory of gravitation. As in any field theory there are two aspects to be considered: the field equations and the variational formulation of them. Undoubtedly, general relativity is the best theory we have for the description of gravitational phenomena. The Einstein field equations were obtained on a phenomenological basis and therefore they exhibit a strong observational agreement. On the other hand, the variational formulation of Einstein field equations provides a systematic way to deal with them. However, this formulation must not be considered as fundamental as the field equations themselves and, in fact, several of the problems of general

\footnotetext{
*tapiens@gmail.com

${ }^{\dagger}$ mujevic@ime.unicamp.br
} 
relativity come from its variational formulation. Just to mention a well known problem, the 'unphysical' non-renormalizability of general relativity is obtained from a dimensional analysis of the Einstein-Hilbert Lagrangian even when the Einstein field equations describe 'physically' sensible situations. Therefore, in order to accommodate the new observational data, not describable in the context of general relativity, it would be better to start by looking for field equations with a better observational agreement than just on the basis of theoretical considerations. Of course, many ad hoc field equations can be conceived to agree with observation and in this case a phenomenological argument, such as that leading from Newtonian gravity to general relativity, is lacking. In the face of this situation, and in spite of the comments above, we must allow our search for the needed modifications to be guided with the help of theoretical considerations.

It has been shown recently [3] that several families of gravitational Lagrangians give rise to the same field equations, namely Einstein field equations with a non-null cosmological constant $\Lambda$. Therefore, these field equations are better suited to accommodate the recent observations concerning a non-null value of $\Lambda$. This 'universality' property was originally observed for Lagrangians depending in an arbitrary way from $\langle R\rangle=g^{\mu \nu} R_{\mu \nu}(\boldsymbol{\Gamma})[18,19,20,21]$. Later on this property was established for a wider family of Lagrangians depending at most on $\left\langle R^{2}\right\rangle=R_{\mu \nu}(\boldsymbol{\Gamma}) R^{\mu \nu}(\boldsymbol{\Gamma})[3]$.

In the present work, we show that these universal field equations can be obtained from a family of variational principles which is even wider than that considered previously. In addition, almost any metric-affine Lagrangian will serve the prupose of providing a variational description of them. Furthermore, we show that, under certain circumstances, it is also possible to incorporate an anisotropy through the appearance of a Weyl vector related to conformal invariance. In previous works this possibility was established only for $n=2$, where $n$ is the dimension of the base space. Here we show that this can be done in any dimension. This result is particularly relevant for $n=4$ since a Weyl vector may describe the recently observed anisotropy of the Universe.

The freedom in the choice of the Lagrangian provides us with a theoretical setting to incorporate almost any other desirable property of a viable gravitational theory, in particular renormalizability and scale invariance, without restricting the field equations.

Let us start with a brief reminder of the state of the art in canonical gravity. A large family of different situations in gravitational physics is correctly described by the Einstein field equations. The problem of obtaining Einstein field equations from a variational principle was first addressed, almost contemporarily, by Einstein [4] and by Hilbert [5]; cf. [6] for an interesting historical account. Under a suitable set of simplifying assumptions they concluded that a possible Lagrangian for the Einstein field equations is given by

$$
\mathcal{L}_{E H}=R(\mathbf{g}) g^{1 / 2},
$$

where $R(\mathbf{g})=g^{\mu \nu} R_{\mu \nu}(\mathbf{g})$ is the Ricci scalar constructed from a purely metric Ricci tensor $R_{\mu \nu}(\mathbf{g})$. Almost inmediately, Palatini [7] presented an alternative Lagrangian yielding the same field equations, namely

$$
\mathcal{L}_{P}=g^{\mu \nu} R_{\mu \nu}(\boldsymbol{\Gamma}) g^{1 / 2},
$$

where $R_{\mu \nu}(\boldsymbol{\Gamma})$ is the symmetric part of the Ricci tensor for a connection $\Gamma^{\lambda}{ }_{\mu \nu}$ symmetric in its two lower indices.

For later convenience we will display our convention for the Ricci tensor. The starting point is the Riemann tensor given by

$$
R_{\rho \mu \nu}^{\lambda}(\boldsymbol{\Gamma})=\partial_{\mu} \Gamma_{\nu \rho}^{\lambda}-\partial_{\nu} \Gamma_{\mu \rho}^{\lambda}+\Gamma_{\mu \sigma}^{\lambda} \Gamma_{\nu \rho}^{\sigma}-\Gamma_{\nu \sigma}^{\lambda} \Gamma_{\mu \rho}^{\sigma},
$$


and its is obviously antisymmetric in its last two indices. From contractions of the Riemann tensor alone, not involving any auxiliary field, two second-rank tensors can be obtained, namely $F_{\mu \nu}=R_{\lambda \mu \nu}^{\lambda}=\partial_{\mu} \Gamma_{\nu}-\partial_{\nu} \Gamma_{\mu}$, with $\Gamma_{\mu}=\Gamma_{\mu \lambda}^{\lambda}$, and the Ricci tensor

$$
R_{\mu \nu}(\boldsymbol{\Gamma})=R_{\mu \lambda \nu}^{\lambda}(\boldsymbol{\Gamma})=\partial_{\lambda} \Gamma_{\nu \mu}^{\lambda}-\partial_{\nu} \Gamma_{\lambda \mu}^{\lambda}+\Gamma_{\lambda \sigma}^{\lambda} \Gamma_{\nu \mu}^{\sigma}-\Gamma_{\nu \sigma}^{\lambda} \Gamma_{\lambda \mu}^{\sigma} .
$$

In the rest of the work we will restrict our considerations to the symmetric part of the Ricci tensor (4) for a connection $\Gamma^{\lambda}{ }_{\mu \nu}$ symmetric in its two lower indices.

The Lagrangians (1) and (2) both lead to the same set of field equations, namely, the Einstein field equations

$$
R_{\mu \nu}(\mathbf{g})-\frac{1}{2} R(\mathbf{g}) g_{\mu \nu}=0 .
$$

After considering the trace of equation (5), which implies $R(\mathbf{g})=0$, the Einstein field equations can be rewritten in the simplified, but equivalent form

$$
R_{\mu \nu}(\mathbf{g})=0 .
$$

The exception is the dimension $n=2$ in which case (5) is an identity. Equations (6) have a strong phenomenological support since, in the non-relativistic limit, they are equivalent to Newtonian gravity and furthermore they have been tested and found to be correct in a variety of situations beyond the realm of Newtonian gravity [8]. However, due to several reasons stemming from the need to obtain better observational agreements [9] to formal problems [10], such as those found in quantum gravity, alternative theories need to be seriously considered.

The first modification of generqal relativity is due to Einstein himself [11] and involves the introduction of a cosmological constant $\Lambda$. In this case, the Lagrangians (1) and (2) are modified to

$$
\mathcal{L}_{E H \Lambda}=\left[R(\mathbf{g})-\Lambda_{n}\right] g^{1 / 2}
$$

and

$$
\mathcal{L}_{P \Lambda}=\left[g^{\mu \nu} R_{\mu \nu}(\boldsymbol{\Gamma})-\Lambda_{n}\right] g^{1 / 2},
$$

where $n$, as in the rest of this work, denotes the dimension of the base space. In both cases the modified field equations are

$$
R_{\mu \nu}(\mathbf{g})-\frac{1}{2}\left[R(\mathbf{g})-\Lambda_{n}\right] g_{\mu \nu}=0 .
$$

Considering the trace of this equation, which implies $R(\mathbf{g})=n \Lambda_{n} /(n-2)$, the Einstein field equations, with a cosmological constant, can be rewritten in the simplified form

$$
R_{\mu \nu}(\mathbf{g})-\frac{1}{(n-2)} \Lambda_{n} g_{\mu \nu}=0 .
$$

The exception is the case for $n=2$ in which the first two terms in (9) are an identity and therefore $\Lambda_{2}=0$. In view of this result it is more convenient to write $\Lambda_{n}=(n-2) \Lambda$. With this new parametrization the field equations (10) are rewritten as

$$
R_{\mu \nu}(\mathbf{g})-\Lambda g_{\mu \nu}=0
$$


and the trace is given simply by $R(\mathbf{g})=n \Lambda$. Our later generalizations will be based on this simplified, but equivalent, form of Einstein field equations.

As mentioned above, recent observations [1] point to a non-null value of the cosmological constant $\Lambda$ and therefore (11), rather than (6), need to be considered as the correct field equations for the gravitational field. A small, but non-null, value of the cosmological constant is most welcome since it is able to solve several observational problems, such as conflicting ages of stars compared with the age of the Universe [12]. The Newtonian limit remains unmodified since $\Lambda$ is small enough to play a significant role at that regime.

The Lagrangians (7) and (8) are correct classically, since they lead to the correct field equations. However, due to several reasons, mainly non-renormalizability, they do not serve as a starting point for a quantization program. As remarked by Martellini [13], what is really relevant for a variational formulation of a gravitational theory is to obtain the correct classical equations, in this case (11), but the Lagrangian is completely unrestricted for the rest and may even strongly differ from the simple prescriptions contained in (7) and (8). This remark is most important when one looks for better starting points for a quantum gravity programme.

The simplest model pointing to satisfy the renormalizability requirements necessary for quantum gravity is the Lagrangian $R^{2}$. In the purely metric case we have

$$
\mathcal{L}_{R^{2}(\mathbf{g})}=\left[\alpha R^{2}(\mathbf{g})+\beta R_{\mu \nu}(\mathbf{g}) R^{\mu \nu}(\mathbf{g})+\gamma R_{\mu \nu \lambda \rho}(\mathbf{g}) R^{\mu \nu \lambda \rho}(\mathbf{g})\right] g^{1 / 2} .
$$

This Lagrangian has been extensively considered from the point of view of necessary counterterms for achieving the renormalizability of the Einstein-Hilbert Lagrangian. However, the Lagrangian $R^{2}$ can be considered by itself, without any reference to general relativity, avoiding in this way the introduction of dimensionful coupling constants [14]. In this case it is possible to establish an equivalence between (12) and general relativity coupled to some kind of matter. However, the Lagrangian (12) is non-polynomial in the metric field and this makes it useless for a quantization programme [13].

The situation is much better for a metric-affine theory where the starting point is the Lagrangian

$$
\mathcal{L}_{R^{2}(\boldsymbol{\Gamma})}=\left[\alpha\langle R\rangle^{2}+\beta\left\langle R^{2}\right\rangle+\gamma \operatorname{Rie}^{2}\right] g^{1 / 2} ;
$$

$\langle R\rangle=g^{\mu \nu} R_{\mu \nu}(\boldsymbol{\Gamma}),\left\langle R^{2}\right\rangle=R_{\mu \nu}(\boldsymbol{\Gamma}) R^{\mu \nu}(\boldsymbol{\Gamma})$, and $\operatorname{Rie}^{2}$ denotes all possible, algebraically independent, contractions of the Riemann tensor $R_{\rho \mu \nu}^{\lambda}(\boldsymbol{\Gamma})$ and since it has fewer symmetries than the RiemannChristoffel tensor $R_{\lambda \rho \mu \nu}(\mathrm{g})$, more terms of that kind need to be considered in (13). In this case it is possible to establish the equivalence between (13) and general relativity with a cosmological constant, as we will shown below. The Lagrangian (13) is far more appealing than (12) since it is polynomial in $\boldsymbol{\Gamma}$ and therefore more amenable for a quantum treatment [13]. The terms $\mathrm{Rie}^{2}$ are Yang-Mills-like and they can be dealt with in that context, see [15]. The terms containing $\langle R\rangle$ and $\left\langle R^{2}\right\rangle$ only, were first considered by Stephenson [16] and by Higgs [17].

Lagrangians with a more general dependence on $\langle R\rangle$ and $\left\langle R^{2}\right\rangle$ have appeared in several studies related to the gravitational field equations. A generic Lagrangian of the form $\mathcal{L}_{\langle R\rangle}=f(\langle R\rangle) g^{1 / 2}$ was considered by Lim [18] and, under a simple assumption, he obtained Einstein field equations with a cosmological constant, namely (11). This was a first indication that one can consider a wider family of variational principles from which to obtain gravitational equations. For a restricted family of Lagrangians $\mathcal{L}_{\langle R\rangle}$, Hamity and Barraco [19] obtained similar field equations in which, in addition to the cosmological constant $\Lambda$, conformal invariance was also incorporated. Finally, Ferraris et al. $[20,21]$ has established the important result that all Lagrangians $\mathcal{L}_{\langle R\rangle}$ possess 'universal' field equations corresponding to Einstein field equations with a cosmological constant $\Lambda$, 
namely (11). Recently, the universality of the field equations (11) was also proved for Lagrangians with an arbitary dependence on $\left\langle R^{2}\right\rangle[3]$.

These results could be interpreted as an indication that the cosmological constant and other desirable properties of the gravitational theory may be obtained as properties of the field equations, rather than being incorporated into the Lagrangian.

In dimension $n=2$, it is possible to incorporate a Weyl vector $W_{\mu}$ related to conformal invariance. The presence of a vector field indicates the existence of a preferred direction. For $n=4 \mathrm{a}$ vector field would be a most welcome ingredient in the field equations since this might describe the recently observed anisotropy of the universe [2].

In the present work we show that the universal field equations (11) can be obtained from a wider family of Lagrangians given by $\mathcal{L}=L(\langle\mathbf{R}\rangle) g^{1 / 2}$, with an arbitrary dependence on $\langle R\rangle^{\mu}{ }_{\nu}=$ $g^{\mu \lambda} R_{\lambda \nu}(\boldsymbol{\Gamma})$. Therefore, it may depend on $\langle R\rangle$ and $\left\langle R^{2}\right\rangle$, but also on higher-order powers of $\langle R\rangle^{\mu}{ }_{\nu}$. More importantly, we show that for any dimension, in particular for $n=4$, under certain circumstances, it is possible to incorporate a Weyl vector field $W_{\mu}$. This is also possible in the situations studied previously by other authors, however, they did not mention this possibility. The Weyl vector field $W_{\mu}$ contributes new terms to the field equations which finally read

$$
\begin{aligned}
R_{\mu \nu}(\mathbf{g})-\frac{1}{4}(n-2)\left(\nabla_{\mu} W_{\nu}+\nabla_{\nu} W_{\mu}\right)+\frac{1}{4}(n-2) W_{\mu} W_{\nu} & \\
-\left(\frac{1}{2}(\nabla W)+\frac{1}{4}(n-2) W^{2}\right) g_{\mu \nu}-\Lambda g_{\mu \nu} & =0,
\end{aligned}
$$

where $(\nabla W)=g^{\mu \nu} \nabla_{\mu} W_{\nu}$ and $W^{2}=g^{\mu \nu} W_{\mu} W_{\nu}$. For the special case $n=4$ the field equations above reduce to

$$
R_{\mu \nu}(\mathbf{g})-\frac{1}{2}\left(\nabla_{\mu} W_{\nu}+\nabla_{\nu} W_{\mu}\right)+\frac{1}{2} W_{\mu} W_{\nu}-\frac{1}{2}\left((\nabla W)+W^{2}\right) g_{\mu \nu}-\Lambda g_{\mu \nu}=0 .
$$

Our method of proof is essentially different from the one used in [3, 20, 21] and is strongly based on a systematic use of the Cayley-Hamilton theorem, which we will now describe.

Let us review some fundamental results related to the Cayley-Hamilton theorem. Let us start by considering a generic $n \times n$ matrix $\langle R\rangle^{\mu}{ }_{\nu}$. Higher powers of this matrix are denoted by

$$
\left\langle R^{k}\right\rangle_{\nu}^{\mu}=\underbrace{\langle R\rangle^{\mu} \cdots\langle R\rangle_{\nu}^{\circ}}_{k \text { times }} .
$$

Accordingly, we denote the traces by

$$
\left\langle R^{k}\right\rangle=\langle R\rangle_{\mu}^{\mu}
$$

Let us introduce next the cumulant coefficients $\left\langle C^{k}\right\rangle$ given by

$$
\begin{aligned}
\left\langle C^{0}\right\rangle & =1 \\
\left\langle C^{1}\right\rangle & =\langle R\rangle \\
\left\langle C^{2}\right\rangle & =\frac{1}{2}\left(\langle R\rangle^{2}-\left\langle R^{2}\right\rangle\right) \\
\left\langle C^{3}\right\rangle & =\frac{1}{3 !}\left(\langle R\rangle^{3}-3\langle R\rangle\left\langle R^{2}\right\rangle+2\left\langle R^{3}\right\rangle\right), \\
\left\langle C^{4}\right\rangle & =\frac{1}{3 !}\left(\langle R\rangle^{4}-6\langle R\rangle^{2}\left\langle R^{2}\right\rangle+8\langle R\rangle\left\langle R^{3}\right\rangle+3\left\langle R^{2}\right\rangle^{2}-6\left\langle R^{4}\right\rangle\right),
\end{aligned}
$$


Essentially the cumulants relate the coefficients in a Taylor series with those of a Fourier expansion. some of the cumulants (18) are familiar objects in matrix calculus; namely, $\left\langle C^{1}\right\rangle=\operatorname{tr}\left(\langle R\rangle^{\mu}{ }_{\nu}\right)$ and $\left\langle C^{n}\right\rangle=\operatorname{det}\left(\langle R\rangle_{\nu}^{\mu}\right)$. A further property of the cumulants is that, for a given $n$, only the first $n$ cumulants are non-trivial, while upper cumulants are identically zero, $\left\langle C^{k}\right\rangle \equiv 0, k>n$.

The Cayley-Hamilton theorem [22] states that only the first $(n-1)$ powers of $\langle R\rangle^{\mu}{ }_{\nu}$ are linearly independent. The relation among them is the characteristic polynomial

$$
\sum_{k=0}^{n}(-)^{k}\left\langle C^{k}\right\rangle\left\langle R^{n-k}\right\rangle_{\nu}^{\mu} \equiv 0 .
$$

Accordingly, only the first $n$ traces (17) are algebraically independent.

For the purposes of this work we introduce a further set of relevant quantities which are obtained by considering derivatives of the cumulants $\left\langle C^{k}\right\rangle$ as follows. Let

$$
\left\langle C^{k}\right\rangle_{\nu}^{\mu}=\left(\frac{\partial\left\langle C^{k}\right\rangle}{\partial\left(\delta_{\mu}^{\nu}\right)}\right)=g^{\mu \lambda} \frac{\partial\left\langle C^{k}\right\rangle}{\partial g^{\lambda \nu}} .
$$

The first $\left\langle C^{k}\right\rangle$ 's are given by

$$
\begin{aligned}
\left\langle C^{1}\right\rangle^{\mu}{ }_{\nu} & =\langle R\rangle^{\mu}{ }_{\nu}, \\
\left\langle C^{2}\right\rangle^{\mu}{ }_{\nu} & =\langle R\rangle\langle R\rangle^{\mu}{ }_{\nu}-\left\langle R^{2}\right\rangle^{\mu}{ }_{\nu}, \\
& =\left\langle C^{1}\right\rangle\left\langle C^{1}\right\rangle^{\mu}{ }_{\nu}-\left\langle C^{1}\right\rangle^{\mu}{ }_{\lambda}\left\langle C^{1}\right\rangle^{\lambda}{ }_{\nu}, \\
\left\langle C^{3}\right\rangle^{\mu}{ }_{\nu} & =\frac{1}{2}\left(\langle R\rangle^{2}-\left\langle R^{2}\right\rangle\right)\langle R\rangle^{\mu}{ }_{\nu}+\left(\left\langle R^{2}\right\rangle^{\mu}{ }_{\lambda}-\langle R\rangle\langle R\rangle^{\mu}{ }_{\lambda}\right)\langle R\rangle_{\nu}^{\lambda}{ }_{\nu} \\
& =\left\langle C^{2}\right\rangle\left\langle C^{1}\right\rangle^{\mu}{ }_{\nu}-\left\langle C^{2}\right\rangle^{\mu}{ }_{\lambda}\left\langle C^{1}\right\rangle^{\lambda}{ }_{\nu}, \\
& \vdots
\end{aligned}
$$

The equation

$$
\left\langle C^{n}\right\rangle^{\mu}{ }_{\nu}=\left\langle C^{n}\right\rangle \delta_{\nu}^{\mu}
$$

is particularly interesting and is a statement similar to (19) but in terms of cumulants. The product of two $\langle C\rangle$ 's follows the rule

$$
\left\langle C^{i}\right\rangle_{\lambda}^{\mu}\left\langle C^{j}\right\rangle_{\nu}^{\lambda}=\sum_{\ell=0}^{k-1}\left[\left\langle C^{i+j-(\ell+1)}\right\rangle\left\langle C^{\ell+1}\right\rangle_{\nu}^{\mu}-\left\langle C^{\ell}\right\rangle_{\lambda}^{\mu}\left\langle C^{i+j-\ell}\right\rangle_{\nu}^{\lambda}\right],
$$

where $\ell=\min (i, j)$. Another interesting relation is given by

$$
\frac{\partial\left\langle C^{k}\right\rangle}{\partial R_{\mu \nu}}=\left\langle C^{k-1}\right\rangle g^{\mu \nu}-\left\langle C^{k-1}\right\rangle^{\mu \nu} .
$$

The relations (21) can be inverted to express $\langle R\rangle$ 's in terms of $\langle C\rangle$ 's. Relations (18) are inverted to 


$$
\begin{aligned}
\langle R\rangle & =\left\langle C^{1}\right\rangle \\
\left\langle R^{2}\right\rangle & =\left\langle C^{1}\right\rangle^{2}-2\left\langle C^{2}\right\rangle \\
\left\langle R^{3}\right\rangle & =\left\langle C^{1}\right\rangle^{3}-3\left\langle C^{1}\right\rangle\left\langle C^{2}\right\rangle+3\left\langle C^{3}\right\rangle \\
& \vdots
\end{aligned}
$$

while relations $(21)$ to

$$
\begin{aligned}
\langle R\rangle^{\mu}{ }_{\nu} & =\left\langle C^{1}\right\rangle^{\mu}{ }_{\nu}, \\
\left\langle R^{2}\right\rangle^{\mu}{ }_{\nu} & =\left\langle C^{1}\right\rangle\left\langle C^{1}\right\rangle^{\mu}{ }_{\nu}-\left\langle C^{2}\right\rangle^{\mu}{ }_{\nu}, \\
\left\langle R^{3}\right\rangle^{\mu}{ }_{\nu} & =\left(\left\langle C^{1}\right\rangle^{2}-\left\langle C^{2}\right\rangle\right)\left\langle C^{1}\right\rangle^{\mu}{ }_{\nu}-\left\langle C^{1}\right\rangle\left\langle C^{2}\right\rangle^{\mu}{ }_{\nu}+\left\langle C^{3}\right\rangle^{\mu}{ }_{\nu}, \\
& \vdots
\end{aligned}
$$

Finally we can write the characteristic polynomial (19) in terms of $\langle C\rangle$ 's just to obtain (22), showing that this is, in fact, a statement equivalent to (19) in terms of the cumulants $\langle C\rangle$ 's.

In view of the above results we are now ready to write the most general Lagrangian with an arbitrary dependence on $\langle R\rangle^{\mu}{ }_{\nu}$. We must consider Lagrangians with a dependence restricted only to the first $n$ traces $\left\langle R^{k}\right\rangle, k=1, \cdots, n$ and this is equivalently done in terms of the $\left\langle C^{k}\right\rangle^{\prime}$ s, $k=1, \cdots, n$. Namely

$$
\mathcal{L}_{n}=L_{n}\left(\left\langle C^{1}\right\rangle, \cdots,\left\langle C^{n}\right\rangle\right) g^{1 / 2} .
$$

Now we must consider a Palatini-like variational principle in which the metric and the connection are varied independently.

Variation with respect to the metric gives

$$
\left\langle C^{n-1}\right\rangle_{\nu}^{\mu} \frac{\partial L_{n}}{\partial\left\langle C^{n-1}\right\rangle}+\cdots+\left\langle C^{1}\right\rangle^{\mu}{ }_{\nu} \frac{\partial L_{n}}{\partial\left\langle C^{1}\right\rangle}+\left(\left\langle C^{n}\right\rangle \frac{\partial L_{n}}{\partial\left\langle C^{n}\right\rangle}-\frac{1}{2} L_{n}\right) \delta_{\nu}^{\mu}=0,
$$

where we have used equation (22). In order to completely determine the tensor $\langle R\rangle^{\mu}{ }_{\nu}$ we need to know its first $(n-1)$ powers and for this purpose it is necessary to have $(n-2)$ other relations similar to (28). We may obtain them by contracting equation (28) with $\left\langle C^{k}\right\rangle^{\mu}{ }_{\nu}, k=1, \cdots, n-2$ and using (22) and (23) to reduce higher powers of $C$ 's. We obtain a set of $n$ relations of the form

$$
\left(\begin{array}{ccc}
D^{1}{ }_{1} & \cdots & D^{1}{ }_{n-1} \\
\vdots & & \vdots \\
D^{n-1}{ }_{1} & \cdots & D^{n-1}{ }_{n-1}
\end{array}\right)\left(\begin{array}{c}
\left\langle C^{1}\right\rangle^{\mu}{ }_{\nu} \\
\vdots \\
\left\langle C^{n-1}\right\rangle^{\mu}{ }_{\nu}
\end{array}\right)=\left(\begin{array}{c}
E^{1} \\
\vdots \\
E^{n-1}
\end{array}\right) \delta_{\nu}^{\mu}
$$

where the coefficients $D$ 's and $E$ 's are functions of $\langle C\rangle$ 's.

Some exceptional cases may appear. The first one is given by the condition $\operatorname{det}(D)=0$, but this is satisfied only for a restricted family of Lagrangians with null measure in the space of functions. Another exceptional situation is obtained when equation (28) is an identity. In this case, no algebraic restrictions are imposed over the Ricci tensor. The solution is

$$
\mathcal{L}_{E}=\sqrt{\left\langle C^{n}\right\rangle} g^{1 / 2}=\sqrt{\operatorname{det}\left[R_{\mu \nu}(\boldsymbol{\Gamma})\right]} .
$$


This is the Eddington Lagrangian [23] which does not depend on the metric, in agreement with the fact that (28) is satisfied identically.

The regular case is characterized by $\operatorname{det}(D) \neq 0$ and we arrive at the relations

$$
\left\langle C^{k}\right\rangle_{\nu}^{\mu}=\left\langle F^{k}\right\rangle \delta_{\nu}^{\mu}
$$

with $\langle F\rangle$ 's functions of $\langle C\rangle$ 's. Therefore, under quite general assumptions the field equations (28) are equivalent to (31). However, this result can be simplified even more since for $k=1$, equation (31) reduces to

$$
\left\langle C^{1}\right\rangle_{\nu}^{\mu}=\langle R\rangle_{\nu}^{\mu}=\left\langle F^{1}\right\rangle \delta_{\nu}^{\mu} .
$$

The cumulants (18) then reduce to

$$
\left\langle C^{k}\right\rangle=\frac{n !}{(n-k) ! k !}\left\langle F^{1}\right\rangle^{k}=\frac{n !}{k !(n-k) !}\left[\frac{1}{n}\langle R\rangle\right]^{k} .
$$

Therefore, equation (32) is rewritten as

$$
R_{\mu \nu}(\boldsymbol{\Gamma})=F(\langle R\rangle) g_{\mu \nu},
$$

where $F(\langle R\rangle)$ is some function of $\langle R\rangle$. The trace of this equation gives $\langle R\rangle=n F(\langle R\rangle)$. Two important situations may appear:

1. If $\langle R\rangle=$ constant, then equation (34) reduces to

$$
R_{\mu \nu}(\boldsymbol{\Gamma})-\Lambda g_{\mu \nu}=0
$$

with $\Lambda=$ constant. Equation (35) above looks the same as the Einstein field equations (11). However, they are not yet equivalent since a metricity condition, i.e., a relationship between $\boldsymbol{\Gamma}$ and $\mathbf{g}$, is still required. This relationship will be obtained starting from equation (46).

2. The second situation appears when the trace of equation (34) is identically zero. This means that (34) must be of the form

$$
R_{\mu \nu}(\boldsymbol{\Gamma})-\frac{1}{n}\langle R\rangle g_{\mu \nu}=0 .
$$

This last situation is the most important for physical applications and we will return to it below.

As a way to verify the correctness of the procedure developed above, let us consider in detail the case $n=3$. The Lagrangian is given by

$$
\mathcal{L}_{3}=L_{3}\left(\left\langle C^{1}\right\rangle,\left\langle C^{2}\right\rangle,\left\langle C^{3}\right\rangle\right) g^{1 / 2} .
$$

Variation with respect to the metric gives

$$
\left\langle C^{1}\right\rangle^{\mu}{ }_{\nu} \frac{\partial L_{3}}{\partial\left\langle C^{1}\right\rangle}+\left\langle C^{2}\right\rangle_{\nu}^{\mu} \frac{\partial L_{3}}{\partial\left\langle C^{2}\right\rangle}+\left(\left\langle C^{3}\right\rangle \frac{\partial L_{3}}{\partial\left\langle C^{3}\right\rangle}-\frac{1}{2} L_{3}\right) \delta_{\nu}^{\mu}=0,
$$

where we have used (22) for $n=3$. The trace of this equation gives 


$$
\left\langle C^{3}\right\rangle \frac{\partial L_{3}}{\partial\left\langle C^{3}\right\rangle}-\frac{1}{2} L_{3}=-\frac{1}{3}\left[2\left\langle C^{2}\right\rangle \frac{\partial L_{3}}{\partial\left\langle C^{2}\right\rangle}+\left\langle C^{1}\right\rangle \frac{\partial L_{3}}{\partial\left\langle C^{1}\right\rangle}\right] .
$$

Therefore, equation (38) is rewritten as

$$
\left\langle C^{1}\right\rangle^{\mu}{ }_{\nu} \frac{\partial L_{3}}{\partial\left\langle C^{1}\right\rangle}+\left\langle C^{2}\right\rangle^{\mu}{ }_{\nu} \frac{\partial L_{3}}{\partial\left\langle C^{2}\right\rangle}-\frac{1}{3}\left[2\left\langle C^{2}\right\rangle \frac{\partial L_{3}}{\partial\left\langle C^{2}\right\rangle}+\left\langle C^{1}\right\rangle \frac{\partial L_{3}}{\partial\left\langle C^{1}\right\rangle}\right] \delta_{\nu}^{\mu}=0 .
$$

Let us now contract this equation with $\left\langle C^{1}\right\rangle^{\mu}{ }_{\nu}$. Using (23) we then obtain

$$
\frac{1}{3}\left[\left\langle C^{2}\right\rangle \frac{\partial L_{3}}{\partial\left\langle C^{2}\right\rangle}+2\left\langle C^{1}\right\rangle \frac{\partial L_{3}}{\partial\left\langle C^{1}\right\rangle}\right]\left\langle C^{1}\right\rangle^{\mu}{ }_{\nu}-\frac{\partial L_{3}}{\partial\left\langle C^{1}\right\rangle}\left\langle C^{2}\right\rangle^{\mu}{ }_{\nu}-\left\langle C^{3}\right\rangle \frac{\partial L_{3}}{\partial\left\langle C^{2}\right\rangle} \delta_{\nu}^{\mu}=0 .
$$

Equations (40) and (41) allow to solve for $\left\langle C^{1}\right\rangle^{\mu}{ }_{\nu}$ and $\left\langle C^{2}\right\rangle^{\mu}{ }_{\nu}$, as in (31), if

$$
\Delta=\frac{1}{3} \frac{\partial L_{3}}{\partial\left\langle C^{2}\right\rangle}\left[\left\langle C^{2}\right\rangle \frac{\partial L_{3}}{\partial\left\langle C^{2}\right\rangle}+2\left\langle C^{1}\right\rangle \frac{\partial L_{3}}{\partial\left\langle C^{1}\right\rangle}\right]+\left(\frac{\partial L_{3}}{\partial\left\langle C^{1}\right\rangle}\right)^{2} \neq 0 .
$$

The condition $\Delta=0$ is fulfilled only by a restricted family of Lagrangians, namely,

$$
L_{3}=\left\langle C^{1}\right\rangle u^{1 / 3}(4 u-1)^{1 / 6}\left(\frac{1+\sqrt{1-3 u}}{1-\sqrt{1-3 u}}\right)^{ \pm 1 / 3}\left(\frac{1+2 \sqrt{1-3 u}}{1-2 \sqrt{1-3 u}}\right)^{\mp 1 / 6} G\left(\left\langle C^{3}\right\rangle\right) .
$$

where $u=\left\langle C^{2}\right\rangle /\left\langle C^{1}\right\rangle^{2}$. Only one sign can be chosen in (43) and $G$ is determined from equation (39). This shows that our derivation is correct, except for a null-measure set.

Let us now turn to the second set of field equations, the metricity condition, which must be obtained from (27). Variation with respect to the connection $\boldsymbol{\Gamma}$ gives

$$
\nabla_{\lambda}\left(\gamma^{\mu \nu} g^{1 / 2}\right)=0
$$

with

$$
\begin{aligned}
\gamma^{\mu \nu} & =\frac{\partial L_{n}}{\partial R_{\mu \nu}} \\
& =g^{\mu \nu} \frac{\partial L_{n}}{\partial\left\langle C^{1}\right\rangle}+\left(\left\langle C^{1}\right\rangle g^{\mu \nu}-\left\langle C^{1}\right\rangle \mu \nu\right) \frac{\partial L_{n}}{\partial\left\langle C^{2}\right\rangle}+\cdots++\left(\left\langle C^{n-1}\right\rangle g^{\mu \nu}-\left\langle C^{n-1}\right\rangle \mu \nu\right) \frac{\partial L_{n}}{\partial\left\langle C^{n}\right\rangle},
\end{aligned}
$$

where we have used (24). This is the Eddington prescription [23] to define a metric in a metricaffine, or purely affine, theory. Let us now consider the two different situations which appeared from (34).

1. Combining equation (45) with equation (35) we obtain $\gamma_{\mu \nu}=K g_{\mu \nu}$, where $K=$ constant. Therefore, $g_{\mu \nu}$ satisfies equation (44), with $\gamma_{\mu \nu}=g_{\mu \nu}$. The solution to (44) is

$$
\Gamma_{\mu \nu}^{\lambda}=\left\{\begin{array}{c}
\lambda \\
\mu \nu
\end{array}\right\}(\mathbf{g})=\frac{1}{2} g^{\lambda \rho}\left(\partial_{\mu} g_{\nu \rho}+\partial_{\nu} g_{\mu \rho}-\partial_{\rho} g_{\mu \nu}\right)
$$

and corresponds to the Christoffel symbol for the metric tensor $g_{\mu \nu}$. Replacing (46) into (35) we obtain the universal field equations (11). The exception is $n=2$ and this case can be considered under the following point. 
2. The second case is much more interesting. In this case the relevant equation is (36). This equation implies that there is a fundamental invariance in the theory. In fact, nothing changes under the transformation $g_{\mu \nu} \rightarrow \Omega(\mathbf{x}) g_{\mu \nu}$. This corresponds to conformal invariance and all of the relevant equations should display this invariance property. Let us remind ourselves that $g^{1 / 2} \rightarrow \Omega^{n / 2} g^{1 / 2}$. Therefore, conformal invariance is guaranteed if $L_{n} \rightarrow \Omega^{-n / 2} L_{n}$. Then $\gamma^{\mu \nu} \rightarrow \Omega^{-n / 2} \gamma^{\mu \nu}$. The relation (44) can be rewritten in a way displaying this property explicitly as

$$
\nabla_{\lambda} \gamma^{\mu \nu}-\frac{1}{n}\left(\gamma_{\sigma \tau} \nabla_{\lambda} \gamma^{\sigma \tau}\right) \gamma^{\mu \nu}=0
$$

With all the ingredients above we can write $\gamma^{\mu \nu}$ as

$$
\gamma^{\mu \nu}=\left(\frac{\langle R\rangle}{n \Lambda}\right)^{n / 2-1} g^{\mu \nu}
$$

This construction procedure forces us to introduce, for dimensional reasons, the constant $\Lambda$ with dimensions $\operatorname{dim}[\Lambda]=L^{-2}$; the factor $n$ is there just for computational convenience.

As a consequence of conformal invariance, equation (47) is traceless with respect to $\gamma_{\mu \nu}$ and, therefore, there is an arbitrariness in the solution of (47). The most general solution of (47), incorporating this arbitrariness, is

$$
\nabla_{\lambda} \gamma_{\mu \nu}=-W_{\lambda}^{\prime} \gamma_{\mu \nu}
$$

This equation can be solved for the connection to obtain

$$
\Gamma_{\mu \nu}^{\lambda}=\left\{\begin{array}{c}
\lambda \\
\mu \nu
\end{array}\right\}(\gamma)+W_{\mu \nu}^{\lambda}\left(\gamma, \mathbf{W}^{\prime}\right),
$$

where $\left\{\begin{array}{c}\lambda \\ \mu \nu\end{array}\right\}(\gamma)$ is the Christoffel symbol (46) for the tensor $\gamma_{\mu \nu}$ and

$$
W_{\mu \nu}^{\lambda}\left(\gamma, \mathbf{W}^{\prime}\right)=\frac{1}{2} \gamma^{\lambda \rho}\left(W_{\mu}^{\prime} \gamma_{\nu \rho}+W_{\nu}^{\prime} \gamma_{\mu \rho}-W_{\rho}^{\prime} \gamma_{\mu \nu}\right)
$$

where $W_{\mu}^{\prime}$ is an arbitrary Weyl vector field [24].

Let us now return to equation (48). This relation can be rewritten as

$$
\gamma_{\mu \nu}=e^{\Psi} g_{\mu \nu}
$$

where $e^{\Psi}$ is a dimensionless function given by

$$
e^{\Psi}=\left(\frac{n \Lambda}{\langle R\rangle}\right)^{n / 2-1}
$$

In terms of this parametrization, the connection is now given by

$$
\Gamma_{\mu \nu}^{\lambda}=\left\{\begin{array}{c}
\lambda \\
\mu \nu
\end{array}\right\}(\mathbf{g})+W_{\mu \nu}^{\lambda}(\mathbf{g}, \mathbf{W}),
$$

where $W_{\mu}=W_{\mu}^{\prime}+\partial_{\mu} \Psi$.

Let us finally note that the resulting theory is conformally invariant. One way of breaking the conformal invariance is to let $\Psi$ acquire a specific value. Due to the conformal invariance, we can fix this value at will, in particular, we can choose $\Psi=0$. In this last case we obtain an effective 
theory for low energies where conformal invariance has been broken. In this case the field equations (36) and (53), evaluated for the connection (54) with $\Psi=0$ are

$$
\begin{aligned}
& R_{\mu \nu}(\mathbf{g})-\frac{(n-2)}{4}\left(\nabla_{\mu} W_{\nu}+\nabla_{\nu} W_{\mu}\right)+\frac{(n-2)}{4} W_{\mu} W_{\nu} \\
& -\frac{1}{n}\left[R(\mathbf{g})-\frac{(n-2)}{2}(\nabla W)+\frac{(n-2)}{4} W^{2}\right] g_{\mu \nu}=0, \\
& R(\mathbf{g})-(n-1)(\nabla W)+\frac{(n-1)(n-2)}{4} W^{2}=n \Lambda,
\end{aligned}
$$

where now $\nabla$ is the usual covariant derivative with respect to the metric $g_{\mu \nu}$. The field equations (55) and (56) are written completely in terms of the Riemannian metric $g_{\mu \nu}$ and therefore can be compared, interpreted and tested in familiar physical situations.

We can now turn to the conclusions. Firstly, we have shown that almost all Lagrangians depending on $\langle R\rangle^{\mu}{ }_{\nu}=g^{\mu \lambda} R_{\lambda \nu}(\boldsymbol{\Gamma})$, in a generic and almost arbitrary way, possess the same 'universal' field equations, namely (11). In the circumstances described under point (2), it is furthermore possible to incorporate a preferred direction. The freedom contained in the choice of the starting Lagrangian may be used to satisfy the several different requirements usually needed in order to reach a consistent quantum version of canonical gravity. For $n=4$ the universal field equations are (15) and they correspond to the usual Einstein field equations with a necessarily non-null cosmological constant and a Weyl vector field $W_{\mu}$. Both of these new properties of the gravitational field equations have strong observational support. Naturally, the next step is to look for solutions of equation (15) in particularly interesting physical situations. $\Lambda$ and $W_{\mu}$ are of cosmological character and their values must be determined from astrophysical observations. Let us first analyse the case $W_{\mu}=0$. Recent observations [1] point to a non-null (even when small) value of the cosmological constant $\Lambda$. A non-null cosmological constant does not have a significant role over solar system scales. In fact, the corresponding spherically symmetric solution is the Kottler metric. The Schwarzschild solution is naturally recovered for $\Lambda=0$ and for small $\Lambda$, which is the actual case, there are no observational effects. However, observational effects may be expected at the level of galactic dynamics [25]. On the other hand, there is some evidence that the universe is not completely isotropic, as assumed in the cosmological principle, and recent observations point to the existence of a preferred direction in the Universe [2]. That direction may be accounted for by the Weyl vector field appearing in (15). The possibility of accommodating the observed anisotropy in the field equations (15) is currently under study with the use of Bianchi spaces (the natural anisotropic extension of FRW spaces). Once the value of $W_{\mu}$ is determined, we can look for other observational effects of $W_{\mu}$. However, and in a way similar to the case of the cosmological constant, we believe that the magnitude of the anisotropy is small as to play a significant role over solar system scales. Therefore, observable effects must be sought at the level of galactic dynamics.

In conclusion, the 'universal field equations' (15) can be considered as a viable generalization of the Einstein field equations of general relativity. Furthermore, almost any metric-affine Lagrangian is a good candidate for this purpose and this additional freedom may serve to accommodate several other requirements.

Let us finally add that the universal field equations (15) are not exclusive to the Lagrangians considered here. They have also been obtained in [26], in the context of the recently developed fourth-rank gravity [27]. 


\section{Acknowledgements}

The authors are grateful to G. Rubilar for calling their attention to a fundamental inconsistency in the original formulation of the work.

\section{References}

[1] J. Ostriker and P. Steinhardt, The observational case for a low-density universe with a nonzero cosmological constant, Nature 377, 600 (1995).

[2] B. Nodland and J. P. Ralston, Indication of anisotropy in electromagnetic propagation over cosmological distances, Phys. Rev. Lett. 78, 3043 (1997).

[3] A. Borowiec, M. Ferraris, M. Francaviglia and I. Volovich, Universality of the Einstein equations for Ricci squared Lagrangians, Class. Quantum Grav. 15, 43 (1998).

[4] A. Einstein, Zur allgemeinem Relativitätstheorie, Preuss. Akad. Wiss. Sitz. 1915, 799 (1915)

[5] D. Hilbert, Die Grundlagen der Physik, Nachr. Ges. Wiss. Göttingen Math. Phys. Kl. 3, 395 (1915).

[6] M. Ferraris, M. Francaviglia and C. Reina, Variational formulation of general relativity from 1915 to 1925 "Palatini's method" discovered by Einstein in 1925, Gen. Rel. Grav. 14, 243 (1982).

[7] A. Palatini, Deduzione invariantiva delle equazioni gravitazionali dal principio de Hamilton, Rend. Circ. Mat. Palermo 43, 203 (1919).

[8] C. M. Will, The confrontation between general relativity and experiment: A 1992 update, Int. J. Mod. Phys. D 1, 13 (1992).

[9] H. C. Arp and T. van Flandern, The case against the big bang, Phys. Lett. A 164, 263 (1992).

[10] B. S. DeWitt, Quantum theory of gravitation. I. The canonical theory, Phys. Rev. 160, 1113 (1967).

[11] A. Einstein, Kosmologische Betrachtungen zur allgemeinen Relativitätstheorie, Preuss. Akad. Wiss. Sitz. 142 (1917).

[12] W. L. Freedman et al., Distance to the Virgo cluster galaxy M100 from Hubble telescope observations of Cepheids, Nature 371, 757 (1994).

[13] M. Martellini, Renormalizability of quantum gravity with cosmological constant, Phys. Rev. Lett. 51, 152 (1983).

[14] R. Utiyama and B. S. DeWitt, Renormalization of a classical gravitational field interacting with quantized matter fields, J. Math. Phys. 3, 608 (1962).

[15] F. W. Hehl, J. D. McCrea, E. W. Mielke and Y. Ne'eman, Metric-affine gauge theory of gravity: Field equations, Noether identities, world spinors, and breaking of dilaton invariance, Phys. Rep. 258, 1 (1995).

[16] G. Stephenson, Quadratic Lagrangians and general relativity, Nuovo Cimento 9, 263 (1958). 
[17] P. W. Higgs, Quadratic Lagrangians and general relativity, Nuovo Cimento 11, 816 (1959).

[18] P. H. Lim, Modification of the Palatini variational principle in general relativity, Phys. Rev. D 27, 719 (1983).

[19] V. H. Hamity and D. E. Barraco, First order formalism of $f(R)$ gravity, Gen. Rel. Grav. 25, 461 (1993).

[20] M. Ferraris, M. Francaviglia and I. Volovich, Universal gravitational equations, Nuovo Cimento B 108, 1313 (1993).

[21] M. Ferraris, M. Francaviglia and I. Volovich, The universality of Eisntein equations with cosmological constant, Class. Quantum Grav. 11, 1505 (1994).

[22] E. D. Nering, Linear Algebra and Matrix Theory (Wiley, New York, 1970).

[23] A. S. Eddington, The Mathematical Theory of Relativity (Cambridge University Press, Cambridge, 1923).

[24] H. Weyl, Space, Time, Matter (Dover, New York, 1926).

[25] P. D. Mannheim, Are galactic rotation curves really flat?, Astrophys. J. 479, 659 (1997).

[26] V. Tapia and D. K. Ross, Conformal fourth-rank gravity, non-vanishing cosmological constant and anisotropy, Class. Quantum Grav. 15, 245 (1998).

[27] V. Tapia, D. K. Ross, A. L. Marrakchi and M. Cataldo, Renormalizable conformally invariant model for the gravitational field, Class. Quantum Grav. 13, 3261 (1996). 